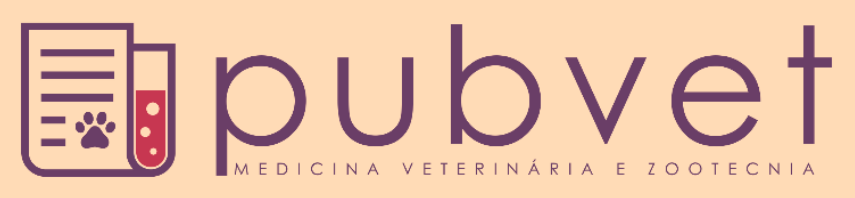

https://doi.org/10.31533/pubvet.v13n01a244.1-5

\title{
Identificação de fungos ungueais em gatos sadios de Santa Catarina de interesse na saúde pública
}

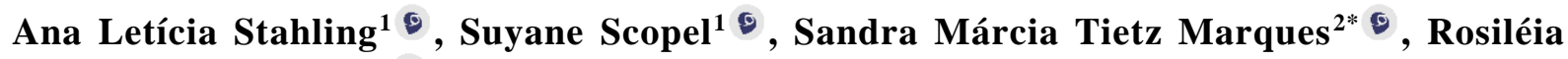 \\ Marinho de Quadros ${ }^{3}$ ( \\ Biomédicas, Técnicas em laboratório, Lages, SC, Brasil. \\ ${ }^{2}$ Dra. Médica Veterinária, Departamento de Patologia Clínica Veterinária, Faculdade de Veterinária, UFRGS, Porto Alegre, RS, Brasil. \\ ${ }^{3}$ Prof. Dra. do Curso de Biomedicina - UNIPLAC, profa. Médica Veterinária da UDESC, Lages, SC. \\ *Autor para Correspondência: Faculdade de Veterinária, Universidade Federal do Rio Grande do Sul, Av. Bento Gonçalves, 9090 , Bairro \\ Agronomia, CEP 90540-000, Porto Alegre, RS. E-mail: sandra.marques@ufrgs.br
}

RESUMO. Objetivou-se avaliar o perfil micológico de interesse na saúde pública de espécies de fungos e a patogenicidade em unhas de 190 gatos de duas cidades de Santa Catarina em 2016. Unhas das patas dianteiras foram coletadas, processadas para exame direto e a cultura mantida a temperatura de $27^{\circ} \mathrm{C}$ durante 15 dias. A prevalência de fungos foi de 83,16\% com identificação dos gêneros Penicillium sp., Aspergillus sp., Fusarium sp., Acremonium sp., Scopulariopsis sp., Curvularia sp., hialohifomicetos, feohifomicetos e Candida albicans. Este é o primeiro registro de fungos de interesse médico em felinos em Santa Catarina. Recomenda-se cautela no manejo de felinos para evitar trauma perfurante e risco de infecção, especialmente para médicos e auxiliares em hospitais veterinários, clínicas e abrigos.

Palavras chave: amostras ungueais, fungos saprófitos, gatos sadios

\section{Identification of nail fungi in healthy cats of Santa Catarina with interest in public health}

\begin{abstract}
The objective of this study was to evaluate mycological profile in public health of fungi species and their pathogenicity in nails of 190 cats from two cities of Santa Catarina in 2016. Nails of front paws were collected, processed for direct examination and the culture was maintained at temperature of $27^{\circ} \mathrm{C}$ for 15 days. The prevalence of fungi was $83.16 \%$ with identification of the genus Penicillium sp., Aspergillus sp., Fusarium sp., Acremonium sp., Scopulariopsis sp., Curvularia sp., hyalohiphomycetes, Phohoifycetes and Candida albicans. This is the first record of fungi of medical interest in felines in Santa Catarina. Caution is recommended in handling felines to avoid perforating trauma and risk of infection, especially veterinarians and their staff in veterinary hospital, clinics and shelters.
\end{abstract}

Keywords: Healthy cats, saprophyte fungi, nail samples

\section{Identificación de hongos en las uñas de gatos sanos de Santa Catarina con interés en la salud publica}

RESUMEN. El objetivo de esta investigación fue evaluar el perfil micológico de interés en la salud pública de especies de hongos y la patogenicidad en uñas de 190 gatos de dos ciudades de Santa Catarina en 2016. Uñas de las patas delanteras fueron colectadas, procesadas para examen directo y la cultura mantenida a temperatura de $27^{\circ} \mathrm{C}$ durante 15 
días. La prevalencia de hongos fue de $83,16 \%$ con identificación de los géneros: Penicillium sp., Aspergillus sp., Fusarium sp., Acremonium sp., Scopulariopsis sp., Curvularia sp., hialohifomicetos, feohifomicetos y Candida albicans. Este es el primer registro de hongos de interés médico en felinos en Santa Catarina. Se recomienda precaución en el manejo de felinos para evitar traumatismo y riesgos de infección, especialmente para médicos y auxiliares en hospitales veterinarios, clínicas y abrigos.

Palabras clave: gatos sanos, hongos saprófitos, muestras de uñas

\section{Introdução}

Com a urbanização e verticalização dos municípios, é observado um aumento no número de adoções de gatos porque eles se adaptam bem em locais menores e, dessa forma, o convívio e o contato direto com o homem também têm aumentado. Os fungos que acometem os animais normalmente pertencem ao grupo dos dermatófitos (geofílicos, zoofílicos ou antropofílicos). As espécies zoofílicas são patógenos preferenciais dos animais, mas também podem causar infecção no homem (Ferreiro et al., 2014). Diversos gêneros de fungos já foram encontrados em decalques de unhas de felinos como Penicillium, Aspergillus, Rhodotorula, Candida, Trichoderma e Acremonium. Os fungos patogênicos e encontrados em unhas são Sporothrix schenkii, Microsporum canis e Malassezia pachydermatis (Borges et al., 2013), além de fungos oportunistas capazes de causar infecções cutâneas, como Alternaria sp., Aspergillus flavus, Curvularia sp., Fusarium sp., Penicillium sp. e Scopulariopsis sp. (Fisher \& Cook 2001; Ferreiro et al., 2014).

A cromoblastomicose é uma infecção crônica subcutânea de seres humanos e animais, cujos agentes etiológicos são introduzidos no tecido por trauma, geralmente através da exposição ocupacional, cujos principais agentes causadores da doença são Fonsecaea pedrosoi, F. compacta, Phialophora verrucosa, Rhinocladiella aquaspersa e Cladosporium carrionii (Sidrim \& Moreira 1999). Entre as principais micoses subcutâneas com envolvimento de felinos destaca-se a esporotricose, onde as lesões principais são decorrentes da formação de úlceras e abscessos ao longo dos canais linfáticos. A maioria das infecções é causada pela inoculação traumática do fungo Sporothrix schenkii nos tecidos do hospedeiro (Sidrim \& Moreira 1999; Fisher \& Cook 2001). A doença vem se tornando um problema de saúde pública no estado do Rio de Janeiro, Brasil, devido à proporção epidêmica nos últimos anos (Barros et al., 2010).

O objetivo deste trabalho foi determinar a presença de fungos em unhas de gatos clinicamente saudáveis dos municípios de Lages e Otacílio Costa, Santa Catarina, região sul do Brasil.

\section{Material e métodos}

A pesquisa incluiu 190 gatos clinicamente sadios, atendidos de março a novembro de 2016, de quatro estabelecimentos: Hospital de Clínica Veterinária do Centro de Ciências Agro veterinárias (CAV) da Universidade do Estado de Santa Catarina (UDESC); Centro de Controle de Zoonoses (CCZ) de Lages; Hospital Veterinário do Centro Universitário FACVEST, animais de uma clínica veterinária particular da cidade de Otacílio Costa, situadas na região serrana do estado de Santa Catarina.

As amostras foram obtidas através da exposição das unhas dos gatos pressionando os tórulos digitais e uma unha direita e esquerda da pata anterior foi cortada com alicate de uso específico, colocadas em microtubos, transportadas em até 24 horas com semeadura em Ágar Sabouraud Dextrose a 2\% acrescido com cloranfenicol e incubadas à temperatura de $27^{\circ} \mathrm{C}$ em um período máximo de 15 dias. As colônias foram avaliadas pelas características de coloração, aspecto, topografia, pigmentação, a presença de hifas, conídios e células leveduriformes pelo exame direto com coloração de azul de lactofenol. Para identificação de fungos do gênero Candida foi utilizado o CHROMagar. Todo processamento do material foi realizado no Laboratório de Cultura da Universidade do Planalto Catarinense (UNIPLAC). Foi usado o teste $\chi 2$ pelo programa R-Core-Team (2016), adotando $\mathrm{P} \leq 0,05$ como nível de significância com intervalo de confiança de $95 \%$ (IC) para as variáveis de gênero, raça, idade e procedência dos animais. A pesquisa foi aprovada pelo comitê de ética em pesquisa em animais do CEUA (Comissão de Ética no Uso de Animais) do CAV-UDESC, com protocolo de número 1.05.15. 


\section{Resultados e discussão}

A prevalência para fungos isolados nas unhas dos gatos foi de 83,16\% (158/190).

Nas unhas da pata direita, 50\% (95/190) pertencem ao grupo de fungos filamentosos hialinos [Penicillium sp. 3,2 \% (3/95), Aspergillus sp. 2,1\% (2/95), Acremonium sp. 1,1\% (1/95), Scopulariopsis sp. $15,8 \%$ (15/95)] e fungos hialohifomicetos não identificados 77,89\% (74/95), além de 8,9\% (17/190) de grupo de filamentosos demáceos [Curvularia sp. 29,4\% (5/17)] e feohifomicetos não identificados $70,6 \%(12 / 17)$ e Candida albicans $[5,3 \%$ (10/190)].

Nas unhas da pata esquerda 55,8\% (106/190) foram identificados fungos filamentosos hialinos [Penicillium sp. 1,9\% (2/106), Aspergillus sp. 3,8\% (4/106), Fusarium sp. 1,9\% (2/106), Scopulariopsis sp. 17,0\% (18/106) e fungos hialohifomicetos não identificados 75,5\% (80/106)], 4,7\% (9/190) de filamentosos demáceos [Curvularia sp. 11,1\% (1/9) e 88,9\% de fungos feohifomicetos não identificados (8/9)], e Candida albicans em 5,8\% (11/190) e 33,7\% (64/190) das amostras sem crescimento. Alguns fungos isolados em amostras ungueais estão representados na Figura 1.
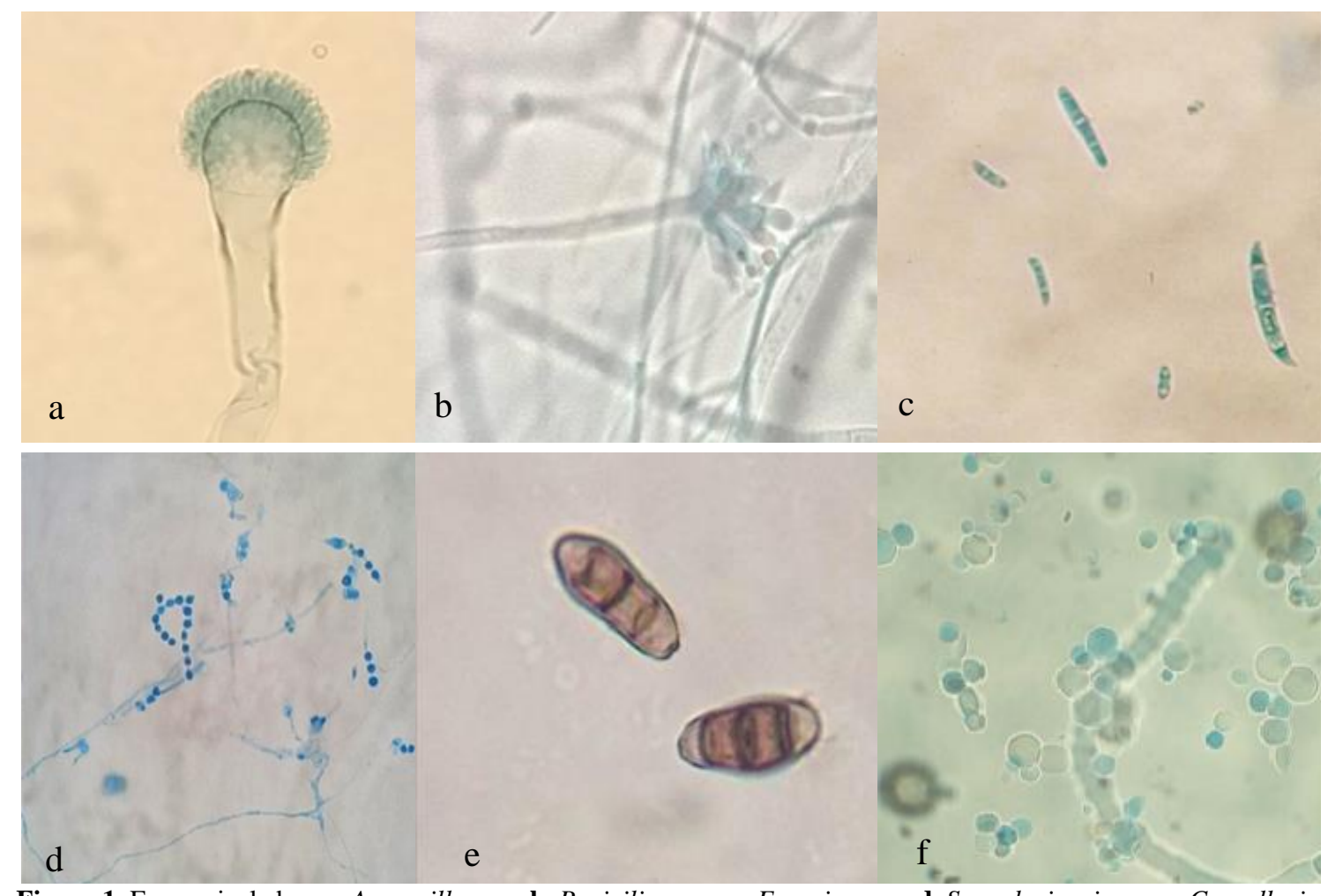

Figura 1. Fungos isolados: a. Aspergillus sp.; b. Penicilium sp.; c. Fusarium sp.; d. Scopulariopsis sp.; e. Curvullaria sp.; f. Candida albicans em amostras ungueais de gatos sadios das cidades de Lages e Otacílio Costa, Santa Catarina em 2016.

Em $83,7 \%$ das fêmeas e $82,6 \%$ dos machos positivo, a maior positividade ocorreu em gatos de até dois anos de idade (36,8\%). Em relação à moradia, 44,2\% (84/190) eram gatos errantes, 28,4\% (54/190), apresentavam proprietários (domiciliados) e 27,4\% (52/190) tinham moradia e acesso livre à rua; $53,1 \%$ (101/190) não eram castrados e 45,8\% (87/190) eram castrados; 90,0\% (171/190) eram sem raça definida; 4,7\% (9/190) persas, 4,7\% (9/190) siameses e 0,5\% (1/190) balinês. As análises estatísticas entre a positividade fúngica dos isolados nas unhas em relação as variáveis de sexo, idade, raça, residência, castração e preferência entre o uso das patas, não houve correção estatística $(P>0,05)$.

A variabilidade de fungos detectados neste estudo ocorreu principalmente em relação aos gatos errantes. Os hábitos sociais da espécie como a procura de fêmeas no cio fazem com que os felinos adultos se aventurem com maior frequência fora de suas residências, como consequência disto haverá um maior contato com o solo. Além disso, a interação entre machos não castrados que buscam por fêmeas para a cópula acaba ocasionando conflitos entre eles (Ferreiro et al., 2014). Esses fatos estão 
diretamente relacionados com o aumento do risco de infecção dos gatos e gera o contato com uma grande variabilidade de fungos que ocorrem no ambiente externo, como os saprofíticos que habitam o solo e vegetais em decomposição e são responsáveis por causar micoses subcutâneas tanto em animais quanto no homem (Cruz 2010). Os gêneros fúngicos Absidia, Mucor, Rhizomucor, Rhizopus e Syncephalastrum também apresentam ampla distribuição geográfica e são isolados do solo e de folhas em decomposição. $\mathrm{Na}$ sua grande maioria são patógenos oportunistas, raramente gerando doença em pacientes saudáveis (Severo et al., 2010). Na infecção fúngica relacionada com indivíduos imunocomprometidos, destacase a feohifomicose, cujos principais agentes são Exophiala jeanselmei e Wangiella dermatitidis, além de vários outros fungos demáceos já recuperados de lesões, como Alternaria sp., Curvularia sp. e Phoma cava (Braga et al., 2013).

Demonstrou-se pela análise da pelagem dos gatos que houve correlação positiva entre crescimento fúngico em gatos com dois anos de idade (animais considerados adultos), sendo que não houve predisposição quanto ao sexo ou raça. Diferentes gêneros de fungos podem estar presentes no solo, dependendo de condições climáticas (temperatura e umidade), $\mathrm{pH}$, presença de nutrientes entre outros fatores (Lima et al., 2016). Deste modo, diferentes tipos de fungos encontrados no solo podem infectar as unhas de gatos, como mostrou esta pesquisa realizada na serra catarinense. Os fungos isolados também foram encontrados em diversos estudos, porém em amostras de pele, como Scopulariopsis sp., Fusarium sp., Curvularia sp., Penicillium sp., Aspergillus sp. e Acremonium sp. encontrados no pelame (Ferreiro et al., 2014).

Todos os fungos encontrados neste trabalho são saprófitas. À vista disso, não se deve descartar a possibilidade de infecção de tecido humano por fungos considerados não patogênicos, principalmente em indivíduos imunocomprometidos. O fungo mais isolado na unha dos gatos desta região catarinense foi o gênero Scopulariopsis, filamentoso e hialino e presente no solo, normalmente é considerado um contaminante e pode provocar infecções em seres humanos, principalmente onicomicose e lesões de pele (Przybysz \& Scolin 2009), portanto com potencial patogênico de causar lesão de pele caso seja inoculado por trauma.

Aspergillus sp. e Penicillium sp. são fungos saprófitas isolados frequentemente no ambiente, assim podem facilmente ser encontrados em amostras de pele de animais, bem como em amostras de unhas de gatos. A respeito de outros gêneros isolados em menor frequência cita-se Acremonium que é capaz de causar fungemia com envolvimento comprovado da pele, demonstrando assim sua capacidade infectante. Outro gênero detectado nas unhas dos gatos foi Curvularia, fungo filamentoso demáceo capaz de causar infecção no trato respiratório, cutâneo e córnea do homem e animais (Taira et al., 2011). Fusarium spp. foi encontrado em apenas duas amostras, porém, é o gênero mais comumente isolado após o Aspergillus sp. em casos de infecção fúngica em pacientes imunocomprometidos, com transmissão através dos tratos respiratório e gastrointestinal, além da pele (Helm et al., 1990).

Entre os fungos mais comumente isolados no Brasil encontra-se o Sporothrix schenkii, causador da esporotricose, uma micose endêmica no Brasil. Este fungo dimórfico é mais ocorrente em condições climáticas tropical e subtropical, apresentando condições de crescimento em temperaturas entre $25^{\circ} \mathrm{C} \mathrm{a}$ $37^{\circ} \mathrm{C}$, podendo causar infecção subaguda ou crônica quando inoculado através de um trauma na pele de humanos ou animais (Fisher \& Cook 2001).

\section{Conclusão}

Neste estudo foi diagnosticada uma variedade de fungos. Diante deste resultado, deve-se ter atenção e cautela, sobretudo aos gatos domésticos que têm acesso à rua, uma vez que estes podem carrear fungos do ambiente em suas unhas e levar ao desenvolvimento de micoses em pessoas, sobretudo imuno comprometidas ou que tenham sido acometidos por algum trauma perfurante, bem como médicos veterinários, enfermeiros e tratadores que atendem grandes grupos de felinos em hospitais, clínicas e Centros de Controle de Zoonoses. É importante salientar que mesmo gatos clinicamente sadios, são capazes de transportar fungos e, dessa forma, há necessidade de adoção de medidas profiláticas para restringir o acesso dos felinos à rua. 


\section{Agradecimento}

Agradecimento especial ao Dr. Paulo Ferrian, professor de Clínica de pequenos animais do CAV/UDESC, por doar parte das amostras para realização deste trabalho e à Médica Veterinária Aline da Rosa Maciel, do Centro de Controle de Zoonoses de Lages, SC.

\section{Referências bibliográficas}

Barros M.B.L., Schubach T.P., Coll J.O., Gremião I.D., Wanke B. \& Schubach A. (2010). Esporotricose: a evolução e os desafios de uma epidemia. Revista Panamericana de Salud Publica 27, 455-460.

Borges, TS., Rossi, C.N., Fedullo, J.D.L, Taborda, J.P. \& Larsson, C. E. (2013). Isolation of Sporothrix schenckii from the claws of domestic cats (indoor and outdoor) and in captivity in São Paulo (Brazil). Mycopathologia, 176, 129-137.

Braga C.A.S.B., Romani A.F., Balestra F.S. \& Ribeiro E.L. (2013). Isolamento de Chaetomium spp. em lesões subcutâneas de um cão\&58; relato de caso. Arquivo Brasileiro de Medicina Veterinária e Zootecnia 65, 37-40.

Cruz L.C.H. (2010). Micologia veterinária. Revinter, Rio de Janeiro.

Ferreiro L., Roehe C., Spanamberg Dorneles A., Machado G., Floriano Fraga C., Gottlieb Lupion C., Javornick Barroso G. \& Cavallini Sanches E.M. (2014). Isolamento de dermatófitos e fungos saprotróficos do pelame de gatos sem dermatoses na região metropolitana de Porto Alegre-RS, Brasil. Acta Scientiae Veterinariae 42.

Fisher F. \& Cook N.B. (2001). Micologia: Fundamentos e Diagnóstico. Revinter Ltda, Rio de Janeiro, Brasil.

Helm T.N., Longworth D.L., Hall G.S., Bolwell B.J., Fernandez B. \& Tomecki K.J. (1990). Case report and review of resolved fusariosis. Journal of the American Academy of Dermatology 23, 393-398.

Lima S.R., Silva W.A., Silveira M.M., Neves R.C.S.M., Dutra V. \& Sousa V.R.F. (2016). Isolamento de dermatófitos em 50 felinos assintomáticos atendidos no HOVET-UFMT. Cuiabá. Semina: Ciências Agrárias 37, 2003-2008.

Przybysz C.H. \& Scolin E. (2009). Avaliação do formaldeído como fungicida no laboratório de anatomia humana.Rev F@ pciência 5, 121-133.

R-Core-Team (2016). R: A language and environment for statistical computing. R. Foundation for Satatical Computing, Viena.

Severo C.B., Guazzelli L.S. \& Severo L.C. (2010). Zygomycosis. Jornal Brasileiro de Pneumologia 36, 134-141.

Sidrim J.J. \& Moreira J.L.B. (1999). Fundamentos Clínicos e Laboratoriais da Micologia Médica. Guanabara Koogan, Rio de Janeiro, Brasil.

Taira C.L., Mota V.A. \& Svidzinski T.I.E. (2011). Virulence potential of filamentous fungi isolated from poultry barns in Cascavel, Paraná, Brazil. Brazilian Journal of Pharmaceutical Sciences 47, $155-160$.

Recebido: 1 novembro, 2018.

Aprovado: 20 novembro, 2018.

Publicado: 11 janeiro, 2019.

Licenciamento: Este artigo é publicado na modalidade Acesso Aberto sob a licença Creative Commons Atribuição 4.0 (CC-BY 4.0), a qual permite uso irrestrito, distribuição, reprodução em qualquer meio, desde que o autor e a fonte sejam devidamente creditados. 